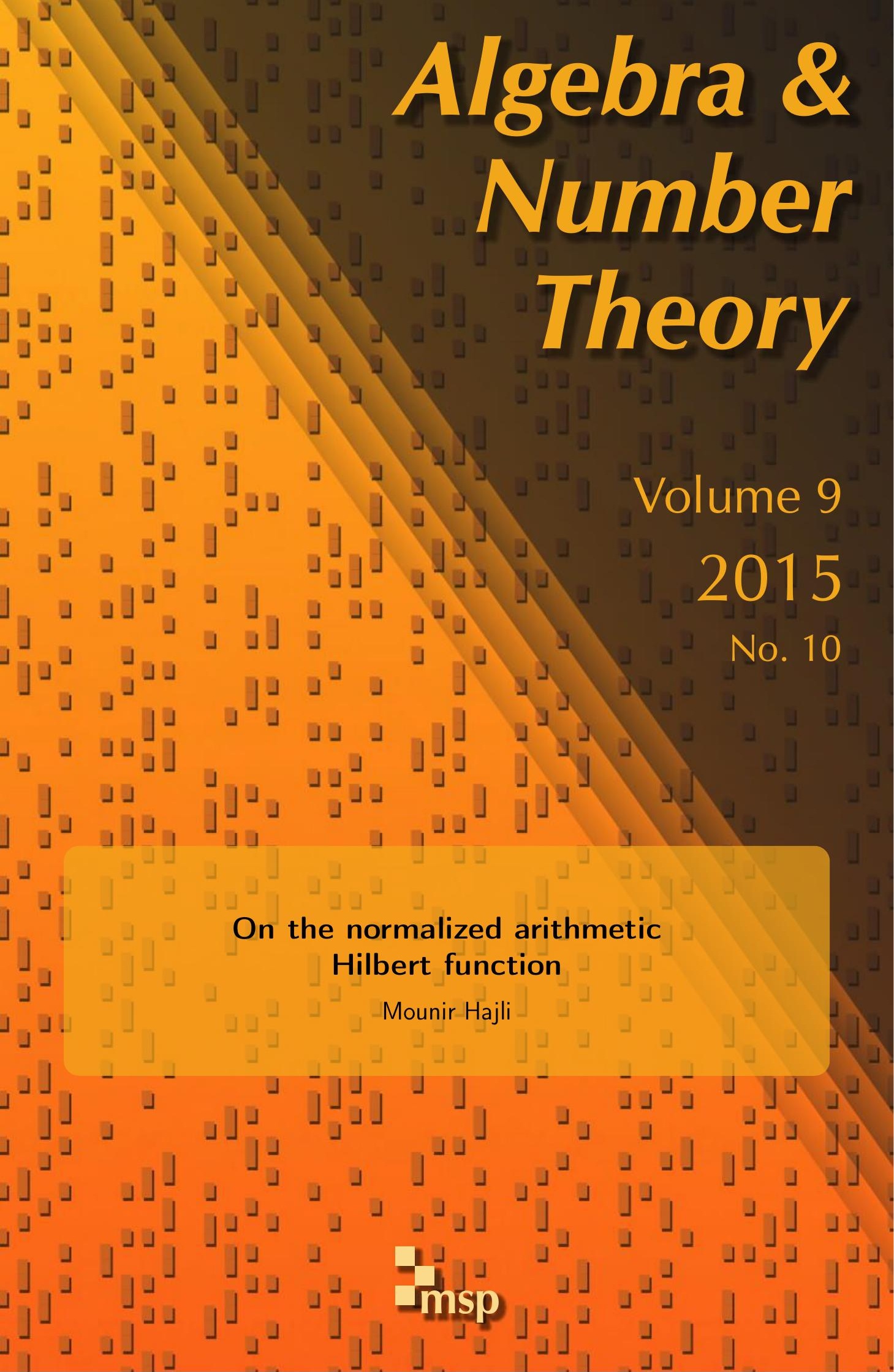




\title{
On the normalized arithmetic Hilbert function
}

\author{
Mounir Hajli
}

Let $X \subset \mathbb{P}_{\overline{\mathbb{Q}}}^{N}$ be a subvariety of dimension $n$, and let $\mathcal{H}_{\text {norm }}(X ; \cdot)$ be the normalized arithmetic Hilbert function of $X$ introduced by Philippon and Sombra. We show that this function admits the asymptotic expansion

$$
\mathcal{H}_{\text {norm }}(X ; D)=\frac{\hat{h}(X)}{(n+1) !} D^{n+1}+o\left(D^{n+1}\right), \quad \forall D \gg 1,
$$

where $\hat{h}(X)$ is the normalized height of $X$. This gives a positive answer to a question raised by Philippon and Sombra.

\section{Introduction}

In [Philippon and Sombra 2008], the authors introduce an arithmetic Hilbert function defined for any subvariety in $\mathbb{P}^{N}$, the projective space of dimension $N$ over $\overline{\mathbb{Q}}$. This function measures the binary complexity of the subvariety. In the case of toric subvarieties, a result of Philippon and Sombra shows that the asymptotic behavior of the associated normalized arithmetic Hilbert function is related to the normalized height of the subvariety considered; see [Philippon and Sombra 2008, Proposition 0.4]. This result is an important step toward the proof of the main theorem of the same paper, which is an explicit formula for the normalized height of projective translated toric varieties; see [Philippon and Sombra 2008, Théorème 0.1].

In [Philippon and Sombra 2008, Question 2.2], the authors ask if the normalized arithmetic Hilbert function admits an asymptotic expansion similar to the toric case. More precisely, given $X$ a subvariety of dimension $n$ in $\mathbb{P}^{N}$, the projective space of dimension $N$ over $\overline{\mathbb{Q}}$, can we find a real $c(X) \geq 0$ such that

$$
\mathcal{H}_{\text {norm }}(X ; D)=\frac{c(X)}{(n+1) !} D^{n+1}+o\left(D^{n+1}\right) ?
$$

If so, do we have $c(X)=\hat{h}(X)$, where $\hat{h}(X)$ is the normalized height of $X$ ?

In this article, we give an affirmative answer to this question.

MSC2010: primary 14G40; secondary 11G50, 11G35.

Keywords: arithmetic Hilbert function, height. 
Theorem 1.1. Let $X \subset \mathbb{P}^{N}$ be a subvariety of dimension $n$ in $\mathbb{P}^{N}$. Then the normalized arithmetic Hilbert function associated to $X$ admits the asymptotic expansion

$$
\mathcal{H}_{\text {norm }}(X ; D)=\frac{\hat{h}(X)}{(n+1) !} D^{n+1}+o\left(D^{n+1}\right), \quad \forall D \gg 1 .
$$

The notion of normalized height plays an important role in the diophantine approximation on tori, particularly in Bogomolov's and generalized Lehmer's problems; see [David and Philippon 1999; Amoroso and David 2003]. A result of Zhang [1992] shows that a subvariety $X$ with a vanishing normalized height is necessarily a union of toric subvarieties.

Gillet and Soulé [1992] proved an arithmetic Hilbert-Samuel formula as a consequence of the arithmetic Riemann-Roch theorem. Roughly speaking, this formula describes the asymptotic behavior of the arithmetic degree of a hermitian module defined by the global sections of the tensorial power of a positive hermitian line bundle on an arithmetic variety. Moreover, the leading term is given by the arithmetic degree of the hermitian line bundle. Later Abbès and Bouche [1995] gave a new proof for this result without using the arithmetic Riemann-Roch theorem. Randriambololona [2006] extended the result Gillet and Soulé to the case of coherent sheaf provided as a subquotient of a metrized vector bundle on an arithmetic variety.

Notation. Let $\mathbb{Q}$ be the field of rational numbers, $\mathbb{Z}$ the ring of integers, $K$ a number field and $\mathcal{O}_{K}$ its ring of integers. For $N$ and $D$ two integers in $\mathbb{N}$ we define $\mathbb{N}_{D}^{N+1}:=\left\{a \in \mathbb{N}^{N+1}: a_{0}+\cdots+a_{N}=D\right\}$, and we let $\mathbb{C}\left[x_{0}, \ldots, x_{N}\right]_{D}$ (resp. $K\left[x_{0}, \ldots, x_{N}\right]_{D}$ ) denote the complex vector space (resp. $K$-vector space) of homogeneous polynomials of degree $D$ in $\mathbb{C}\left[x_{0}, \ldots, x_{N}\right]$ (resp. in $K\left[x_{0}, \ldots, x_{N}\right]$ ).

For any prime number $p$ we denote by $|\cdot|_{p}$ the $p$-adic absolute value on $\mathbb{Q}$ such that $|p|_{p}=p^{-1}$ and by $|\cdot|_{\infty}$, or simply $|\cdot|$, the standard absolute value. Let $M_{\mathbb{Q}}$ be the set of these absolute values. We denote by $M_{K}$ the set of absolute values of $K$ extending the absolute values of $M_{\mathbb{Q}}$, and by $M_{K}^{\infty}$ the subset in $M_{K}$ of archimedean absolute values.

We denote by $\mathbb{P}^{N}$ the projective space over $\overline{\mathbb{Q}}$ of dimension $N$. A variety is assumed reduced and irreducible.

\section{The proof of Theorem 1.1}

We keep the same notation as in [Philippon and Sombra 2008]. Let $\omega$ be the FubiniStudy form on $\mathbb{P}^{N}(\mathbb{C})$. For any $k \in \mathbb{N}_{\geq 1} \cup\{\infty\}$, we denote by $h_{k}$ the hermitian metric on $\mathcal{O}(1)$ given by

$$
h_{k}(\cdot, \cdot)=\frac{|\cdot|^{2}}{\left(\left|x_{0}\right|^{2 k}+\cdots+\left|x_{N}\right|^{2 k}\right)^{1 / 2 k}} \quad \text { and } \quad h_{\infty}(\cdot, \cdot)=\frac{|\cdot|^{2}}{\max \left(\left|x_{0}\right|, \ldots,\left|x_{N}\right|\right)^{2}}
$$


and we let $\overline{\mathcal{O}(1)}_{k}:=\left(\mathcal{O}(1), h_{k}\right)$ and $\omega_{k}:=c_{1}\left(\mathcal{O}(1), h_{k}\right)$ for any $k \in \mathbb{N} \cup\{\infty\}$. Note that $\omega_{k}=(1 / k)[k]^{*} \omega$, where $[k]: \mathbb{P}^{N}(\mathbb{C}) \rightarrow \mathbb{P}^{N}(\mathbb{C}),\left[x_{0}: \cdots: x_{N}\right] \mapsto\left[x_{0}^{k}: \cdots: x_{N}^{k}\right]$. Observe that the sequence $\left(\omega_{k}\right)_{k \in \mathbb{N}_{\geq 1}}$ converges weakly to the current $\omega_{\infty}$. We consider the normalized volume form

$$
\Omega_{k}:=\omega_{k}^{\wedge N}, \quad \forall k \in \mathbb{N}_{\geq 1} \cup\{\infty\} .
$$

For any $k \in \mathbb{N}_{\geq 1} \cup\{\infty\}$, the metrics of $\overline{\mathcal{O}(1)}_{k}$ and $\Omega_{k}$ define a scalar product $\mathbb{C}\left[x_{0}, \ldots, x_{N}\right]_{D}$ denoted by $\langle\cdot, \cdot\rangle_{k}$ and given by

$$
\langle f, g\rangle_{k}:=\int_{\mathbb{P}^{N}(\mathbb{C})} h_{k}^{\otimes D}(f, g) \Omega_{k},
$$

for any $f=\sum_{a} f_{a} x^{a}, g=\sum_{a} g_{a} x^{a}$ in $\mathbb{C}\left[x_{0}, \ldots, x_{N}\right]_{D}$ with $f_{a}, g_{a} \in \mathbb{C}$. We denote by $\|\cdot\|_{k}$ the associated norm for any $k \in \mathbb{N}_{\geq 1} \cup\{\infty\}$. Note that $\langle f, g\rangle_{\infty}=\sum_{a} f_{a} \bar{g}_{a}$ and $\left\|x^{a}\right\|_{\infty}=1$ for any $a \in \mathbb{N}_{D}^{N+1}$ and $D \in \mathbb{N}$.

Let $X \subset \mathbb{P}^{N}$ be a subvariety defined over a number field $K$. Define an embedding $\sigma_{v}: K \rightarrow \mathbb{C}$, where $v \in M_{K}^{\infty}$. For any $p_{1}, \ldots, p_{l} \in K\left[x_{0}, \ldots, x_{N}\right]_{D}$, we set

$$
\left\|p_{1} \wedge \cdots \wedge p_{l}\right\|_{k, v}:=\left\|\sigma_{v}\left(p_{1}\right) \wedge \cdots \wedge \sigma_{v}\left(p_{l}\right)\right\|_{k}, \quad \forall k \in \mathbb{N} \cup\{\infty\} .
$$

Define $\mathcal{O}(D):=\mathcal{O}(1)^{\otimes D}$. We let $M:=\Gamma\left(\Sigma, \mathcal{O}(D)_{\left.\right|_{\Sigma}}\right)$ be the $\mathcal{O}_{K}$-module of global sections of $\mathcal{O}(D)_{\left.\right|_{\Sigma}}$, where $\Sigma$ is the Zariski closure of $X$ in $\mathbb{P}_{\mathcal{O}_{K}}^{N}$. For any $v \in M_{K}^{\infty}$, we set $\Gamma\left(\Sigma, \mathcal{O}(D)_{\left.\right|_{\Sigma}}\right)_{\sigma_{v}}:=\Gamma\left(\Sigma, \mathcal{O}(D)_{\left.\right|_{\Sigma}}\right) \otimes_{\sigma_{v}} \mathbb{C}$. We consider the following restriction map:

$$
\pi: \Gamma\left(\mathbb{P}_{\mathcal{O}_{K}}^{N}, \mathcal{O}(D)\right)_{\sigma_{v}} \rightarrow \Gamma\left(\Sigma, \mathcal{O}(D)_{\left.\right|_{\Sigma}}\right)_{\sigma_{v}} \rightarrow 0 .
$$

The space $\Gamma\left(\mathbb{P}_{\mathcal{O}_{K}}^{N}, \mathcal{O}(D)\right)_{\sigma_{v}}$ is identified canonically to $K_{\sigma}\left[x_{0}, \ldots, x_{N}\right]_{D}$. For any $k \in \mathbb{N}_{\geq 1} \cup\{\infty\}$, this space can be endowed by the scalar product induced by $\Omega_{k}$ and $h_{k}$ and denoted by $\langle\cdot, \cdot\rangle_{k, v}$, where

$$
\langle f, g\rangle_{k, v}=\left\langle\sigma_{v}(f), \sigma_{v}(g)\right\rangle_{k}
$$

for any $f, g \in \Gamma\left(\mathbb{P}_{\mathcal{O}_{K}}^{N}, \mathcal{O}(D)\right)_{\sigma_{v}}$. Since $\mathcal{O}(1)$ is ample, there exists a $D_{0} \in \mathbb{N}$ such that, for any $D \geq D_{0}$, the restriction map is surjective. Let $D \geq D_{0}$. For any $k \in \mathbb{N} \cup\{\infty\}$, we denote by $\|\cdot\|_{k, v, \text { quot }}$ the quotient norm induced by $\pi$ and $\|\cdot\|_{k, v}$. Following [Philippon and Sombra 2008, p. 348], we endow $\Gamma\left(\Sigma, \mathcal{O}(D)_{\left.\right|_{\Sigma}}\right)_{\sigma_{v}}$ with $\|\cdot\|_{k, v \text {,quot }}$, for any $k \in \mathbb{N}_{\geq 1} \cup\{\infty\}$. By this construction, $M$ can be equipped with a structure of a hermitian $\mathcal{O}_{K}$-module, denoted by $\bar{M}_{k}$. If $f_{1}, \ldots, f_{s} \in M$, is a $K$-basis for $M \otimes_{\mathcal{O}_{K}} K$, then

$$
\begin{aligned}
\widehat{\operatorname{deg}}\left(\bar{M}_{k}\right) & \left.=\widehat{\operatorname{deg}}\left(\overline{\Gamma\left(\Sigma, \mathcal{O}(D)_{\left.\right|_{\Sigma}}\right.}\right)_{k}\right) \\
& :=\frac{1}{[K: \mathbb{Q}]}\left(\log \operatorname{Card}\left(\bigwedge^{s} M /\left(f_{1} \wedge \cdots \wedge f_{s}\right)\right)-\sum_{v: K \rightarrow \mathbb{C}} \log \left\|f_{1} \wedge \cdots f_{s}\right\|_{k, v}\right) .
\end{aligned}
$$


The normalized arithmetic Hilbert function. Let $X \subset \mathbb{P}^{N}$ be a subvariety defined over a number field $K$ and let $I:=I(X) \subset K\left[x_{0}, \ldots, x_{N}\right]$ be its ideal of definition. We set

$$
\mathcal{H}_{\text {geom }}(X ; D):=\operatorname{dim}_{K}\left(K\left[x_{0}, \ldots, x_{N}\right] / I\right)_{D}=\left(\begin{array}{c}
D+N \\
N
\end{array}\right)-\operatorname{dim}_{K}\left(I_{D}\right) .
$$

The function $\mathcal{H}_{\text {geom }}(X ; \cdot)$ is known as the classical geometric Hilbert function. Philippon and Sombra [2008] introduced an arithmetic analogue of this function. Define $m:=\mathcal{H}_{\text {geom }}(X ; D), l:=\operatorname{dim}_{K}\left(I_{D}\right)$ and let

$$
\bigwedge^{l} K\left[x_{0}, \ldots, x_{N}\right]_{D}
$$

be the $l$-th exterior power product of $K\left[x_{0}, \ldots, x_{N}\right]_{D}$. For $f \in \bigwedge^{l} K\left[x_{0}, \ldots, x_{N}\right]_{D}$ and $v \in M_{K}$ we denote by $|f|_{v}$ the sup-norm of the coefficients of $f$ at the place $v$, with respect to the standard basis of $\bigwedge^{l} K\left[x_{0}, \ldots, x_{N}\right]_{D}$.

Definition 2.1 [Philippon and Sombra 2008, Définition 2.1]. Let $p_{1}, \ldots, p_{l}$ be a $K$-basis of $I_{D}$. We set

$$
\mathcal{H}_{\text {norm }}(X ; D)=\sum_{v \in M_{K}} \frac{\left[K_{v}: \mathbb{Q}_{v}\right]}{[K: \mathbb{Q}]} \log \left|p_{1} \wedge \cdots \wedge p_{m}\right|_{v} .
$$

By the product formula, this definition does not depend on the choice of the basis; also it is invariant under finite extensions of $K$. We call $\mathcal{H}_{\text {norm }}(X ; \cdot)$ the normalized arithmetic Hilbert function of $X$.

Following Philippon and Sombra, this arithmetic Hilbert function measures, for any $D \in \mathbb{N}$, the binary complexity of the $K$-vector space of forms of degree $D$ in $K\left[x_{0}, \ldots, x_{N}\right]$ modulo $I$. As pointed out by Philippon and Sombra [2008, Proposition 0.4], when $X$ is a toric variety, the asymptotic behavior of its associated normalized arithmetic Hilbert function is related to $\hat{h}(X)$, the normalized height of $X$. The authors ask the following question:

Given $X$ a subvariety in $\mathbb{P}^{N}$ of dimension $n$, can we find a real $c(X) \geq 0$ such that

$$
\mathcal{H}_{\text {norm }}(X ; D)=\frac{c(X)}{(n+1) !} D^{n+1}+o\left(D^{n+1}\right) ?
$$

If so, do we have $c(X)=\hat{h}(X)$ ?

We recall the following proposition, which gives a dual formulation for $\mathcal{H}_{\text {norm }}$. Proposition 2.2. Let $q_{1}, \ldots, q_{m} \in K\left[x_{0}, \ldots, x_{N}\right]_{D}^{\vee}$ be a $K$-basis of $\operatorname{Ann}\left(I_{D}\right)$. Then

$$
\mathcal{H}_{\text {norm }}(X ; D)=\sum_{v \in M_{K}} \frac{\left[K_{v}: \mathbb{Q}_{v}\right]}{[K: \mathbb{Q}]} \log \left|q_{1} \wedge \cdots \wedge q_{m}\right|_{v} .
$$

Proof. See [Philippon and Sombra 2008, Proposition 2.3]. 
For any $k \in \mathbb{N}_{\geq 1} \cup\{\infty\}$, we consider the arithmetic function

$$
\mathcal{H}_{\text {arith }}(X ; D, k)
$$

$$
\begin{aligned}
:=\sum_{v \in M_{K}^{\infty}} & \frac{\left[K_{v}: \mathbb{Q}_{v}\right]}{[K: \mathbb{Q}]} \log \left\|p_{1} \wedge \cdots \wedge p_{l}\right\|_{k, v} \\
& +\sum_{v \in M_{K} \backslash M_{K}^{\infty}} \frac{\left[K_{v}: \mathbb{Q}_{v}\right]}{[K: \mathbb{Q}]} \log \left|p_{1} \wedge \cdots \wedge p_{l}\right|_{v}+\frac{1}{2} \log (\gamma(N, D, k)),
\end{aligned}
$$

where $p_{1}, \ldots, p_{l}$ is a $K$-basis of $I_{D}$ and

$$
\gamma(N ; D, k):=\prod_{a \in \mathbb{N}_{D}^{N+1}}\langle a, a\rangle_{k}^{-1} .
$$

For $k=1$, notice that $\mathcal{H}_{\text {arith }}(X ; \cdot, 1)$ corresponds, up to a constant, to the arithmetic function $\mathcal{H}_{\text {arith }}(X ; \cdot)$ considered in [Philippon and Sombra 2008, p. 346].

Similarly to $\mathcal{H}_{\text {norm }}$, the function $\mathcal{H}_{\text {arith }}$ admits a dual formulation. The scalar product $\langle\cdot, \cdot\rangle_{k}$ induces the following linear isomorphism:

$$
\eta_{k}: \mathbb{C}\left[x_{0}, \ldots, x_{N}\right] \rightarrow \mathbb{C}\left[x_{0}, \ldots, x_{N}\right]^{\vee}, \quad f \mapsto\langle\cdot, f\rangle_{k} .
$$

Thus $\mathbb{C}\left[x_{0}, \ldots, x_{N}\right]^{\vee}$ can be endowed with the dual scalar product, given by

$$
\left\langle\eta_{k}(f), \eta_{k}(g)\right\rangle_{k}:=\langle f, g\rangle_{k}, \quad \forall f, g \in \mathbb{C}\left[x_{0}, \ldots, x_{N}\right]_{D} .
$$

We can check easily that, for any $k \in \mathbb{N} \cup\{\infty\}$, we have

$$
\|\theta\|_{k}^{\prime}:=\sup _{g \in \mathbb{C}\left[x_{0}, \ldots, x_{N}\right] \backslash\{0\}} \frac{|\theta(g)|}{\|g\|_{k}}=\|f\|_{k},
$$

where $f \in \mathbb{C}\left[x_{0}, \ldots, x_{N}\right]$ is such that $\theta=\eta_{k}(f)$. Then $\|\theta\|_{k}^{\prime 2}=\langle\theta, \theta\rangle_{k}$ for any $\theta \in \mathbb{C}\left[x_{0}, \ldots, x_{N}\right]^{\vee}$. It follows that

$$
\langle\theta, \zeta\rangle_{k}=\sum_{b}\left\langle x^{b}, x^{b}\right\rangle_{k}^{-1} \theta_{b} \bar{\zeta}_{b}
$$

This product extends to $\wedge^{m}\left(\mathbb{C}\left[x_{0}, \ldots, x_{N}\right]_{D}^{\vee}\right)$ as follows:

$$
\left\langle\theta_{1} \wedge \cdots \wedge \theta_{m}, \zeta_{1} \wedge \cdots \wedge \zeta_{m}\right\rangle_{k}:=\operatorname{det}\left(\left\langle\theta_{i}, \zeta_{j}\right\rangle_{k}\right)_{1 \leq i, j \leq m}
$$

Proposition 2.3. Let $q_{1}, \ldots, q_{m} \in K\left[x_{0}, \ldots, x_{N}\right]_{D}^{\vee}$ be a $K$-basis of $\operatorname{Ann}\left(I_{D}\right)$. Then

$$
\mathcal{H}_{\text {arith }}(X ; D, k)
$$

$$
=\sum_{v \in M_{K}^{\infty}} \frac{\left[K_{v}: \mathbb{Q}_{v}\right]}{[K: \mathbb{Q}]} \log \left\|q_{1} \wedge \cdots \wedge q_{m}\right\|_{k, v}^{\vee}+\sum_{v \in M_{K} \backslash M_{K}^{\infty}} \frac{\left[K_{v}: \mathbb{Q}_{v}\right]}{[K: \mathbb{Q}]} \log \left|q_{1} \wedge \cdots \wedge q_{m}\right|_{v} .
$$


Proof. The proof is similar to [Philippon and Sombra 2008, Proposition 2.5].

Lemma 2.4. There exists a $D_{1}$ such that, for any $D \geq D_{1}$ and any $k \in \mathbb{N}$, we have

$$
\left.\mathcal{H}_{\text {arith }}(X ; D, k)=\widehat{\operatorname{deg}}\left(\overline{\Gamma\left(\Sigma, \mathcal{O}(D)_{\left.\right|_{\Sigma}}\right.}\right)_{k}\right)-\frac{1}{2} \mathcal{H}_{\text {geom }}(X ; D) \log \left(\begin{array}{c}
D+N \\
N
\end{array}\right) .
$$

Proof. The proof is similar to [Philippon and Sombra 2008, Lemme 2.6]. Let $\mathcal{I}$ be the ideal sheaf of $\Sigma$ and let $\Gamma\left(\mathbb{P}_{\mathcal{O}_{K}}^{N}, \mathcal{I} \mathcal{O}(D)\right)$ be the $\mathcal{O}_{K}$-module of global sections of $\mathcal{I O}(D)$, endowed with the scalar products induced by the scalar product $\langle\cdot, \cdot\rangle_{k}$. We claim that there exists an integer $D_{1}$, which does not depend on $k$, such that, for any $D \geq D_{1}$, we have

$$
\left.\widehat{\operatorname{deg}}\left(\overline{\Gamma\left(\Sigma, \mathcal{O}(D)_{\left.\right|_{\Sigma}}\right.}\right)_{k}\right)=\widehat{\operatorname{deg}}\left(\overline{\Gamma\left(\mathbb{P}_{\mathcal{O}_{K}}^{N}, \mathcal{O}(D)\right)_{k}}\right)-\widehat{\operatorname{deg}}\left(\overline{\Gamma\left(\mathbb{P}_{\mathcal{O}_{K}}^{N}, \mathcal{I O}(D)\right)_{k}}\right)
$$

Indeed, we can find a $D_{1} \in \mathbb{N}$ such that, for all $D \geq D_{1}$, the following sequence is exact:

$$
0 \rightarrow \Gamma\left(\mathbb{P}_{\mathcal{O}_{K}}^{N}, \mathcal{I} \mathcal{O}(D)_{\left.\right|_{\Sigma}}\right) \rightarrow \Gamma\left(\mathbb{P}_{\mathcal{O}_{K}}^{N}, \mathcal{O}(D)\right) \rightarrow \Gamma\left(\Sigma, \mathcal{O}(D)_{\left.\right|_{\Sigma}}\right) \rightarrow 0
$$

Then by [Randriambololona 2001, Lemme 2.3.6], the sequence of hermitian $\mathcal{O}_{K^{-}}$ modules

$$
0 \rightarrow \overline{\Gamma\left(\mathbb{P}_{\mathcal{O}_{K}}^{N}, \mathcal{I O}(D)_{\left.\right|_{\Sigma}}\right)_{k}} \rightarrow \overline{\Gamma\left(\mathbb{P}_{\mathcal{O}_{K}}^{N}, \mathcal{O}(D)\right)_{k}} \rightarrow \overline{\Gamma\left(\Sigma, \mathcal{O}(D)_{\left.\right|_{\Sigma}}\right)_{k}} \rightarrow 0
$$

is exact, where the metrics of $\overline{\Gamma\left(\mathbb{P}_{\mathcal{O}_{K}}^{N}, \mathcal{I O}(D)_{\left.\right|_{\Sigma}}\right)_{k}}$ and $\overline{\Gamma\left(\Sigma, \mathcal{O}(D)_{\left.\right|_{\Sigma}}\right)_{k}}$ are induced by the metric of $\overline{\Gamma\left(\mathbb{P}_{\mathcal{O}_{K}}^{N}, \mathcal{O}(D)\right)_{k}}$.

We have

$$
\widehat{\operatorname{deg}}\left(\overline{\Gamma\left(\mathbb{P}_{\mathcal{O}_{K}}^{N}, \mathcal{O}(D)\right)_{k}}\right)=\frac{1}{2} \log (\gamma(N ; D, k))+\frac{1}{2}\left(\begin{array}{c}
D+N \\
N
\end{array}\right) \log \left(\begin{array}{c}
N+D \\
N
\end{array}\right) .
$$

As in the proof of [Philippon and Sombra 2008, Lemme 2.6], and keeping the same notation, we have

$$
\begin{aligned}
&\left.\widehat{\operatorname{deg}}\left(\overline{\Gamma\left(\Sigma, \mathcal{O}(D)_{\left.\right|_{\Sigma}}\right.}\right)_{k}\right) \\
&=\frac{1}{2} \log (\gamma(N ; D, k))+\frac{1}{2} \mathcal{H}_{\text {geom }}(X ; D) \log \left(\begin{array}{c}
N+D \\
N
\end{array}\right) \\
&+\sum_{v \in M_{K}^{\infty}} \frac{\left[K_{v}: \mathbb{Q}_{v}\right]}{[K: \mathbb{Q}]} \log \left\|p_{1} \wedge \cdots \wedge p_{l}\right\|_{k, v}^{\vee} \\
& \quad-\frac{1}{[K: \mathbb{Q}]} \log \operatorname{Card}\left(\wedge^{l}\left(I_{\mathcal{O}_{K}}\right) /\left(p_{1} \wedge \cdots \wedge p_{l}\right)\right) .
\end{aligned}
$$


The last term in (6) does not depend on the metric. It is computed in [Philippon and Sombra 2008, p. 349]; we have

$$
\begin{aligned}
\frac{1}{[K: \mathbb{Q}]} \log \operatorname{Card}\left(\wedge^{l}\left(I_{\mathcal{O}_{K}}\right) /\left(p_{1} \wedge\right.\right. & \left.\left.\cdots \wedge p_{l}\right)\right) \\
& =-\sum_{v \in M_{K} \backslash M_{K}^{\infty}} \frac{\left[K_{v}: \mathbb{Q}_{v}\right]}{[K: \mathbb{Q}]} \log \left|p_{1} \wedge \cdots \wedge p_{l}\right|_{v} .
\end{aligned}
$$

By [Randriambololona 2006, Théorème A], we have

$$
\widehat{\operatorname{deg}}\left(\overline{\Gamma\left(\Sigma, \mathcal{O}(D)_{\mid \Sigma}\right)_{k}}\right)=\frac{h_{\overline{\mathcal{O}(1)} k}(X)}{(n+1) !} D^{n+1}+o\left(D^{n+1}\right), \quad \forall D \gg 1,
$$

where $h_{\overline{\mathcal{O}(1)_{k}}}(X)$ denotes the height of the Zariski closure of $X$ in $\mathbb{P}_{\mathcal{O}_{K}}^{N}$ with respect to $\overline{\mathcal{O}(1)}_{k}$. Since $\frac{1}{2} \mathcal{H}_{\text {geom }}(X ; D) \log \left(\begin{array}{c}D+N \\ N\end{array}\right)=o\left(D^{n+1}\right)$ for $D \gg 1$, by Lemma 2.4 , we get

$$
\mathcal{H}_{\text {arith }}(X ; D, k)=\frac{h_{\overline{\mathcal{O}(1)} k}(X)}{(n+1) !} D^{n+1}+o\left(D^{n+1}\right), \quad \forall D \gg 1 .
$$

Let $q_{1}, \ldots, q_{m} \in K\left[x_{0}, \ldots, x_{N}\right]^{\vee}$ be a $K$-basis of $\operatorname{Ann}\left(I_{D}\right)$. For any finite subset $M$ in $\mathbb{N}_{D}^{N+1}$ of cardinal $m$, we set $q_{M}:=\left(q_{j b}\right)_{1 \leq j \leq m, b \in M} \in K^{m \times m}$ where the $q_{j b}$ are such that $q_{j}=\sum_{b \in \mathbb{N}_{D}^{N+1}} q_{j b}\left(x^{b}\right)^{\vee}$. For any $v \in M_{K}^{\infty}$, we have

$$
\begin{aligned}
\left|q_{1} \wedge \cdots \wedge q_{m}\right|_{v} & =\max \left\{\left|\operatorname{det}\left(q_{M}\right)\right|_{v}: M \subset \mathbb{N}_{D}^{N+1}, \operatorname{Card}(M)=m\right\} \\
& \leq\left(\sum_{M ; \operatorname{Card}(M)=m}\left(\prod_{b \in M}\langle b, b\rangle_{v, k}^{-1}\right)\left|\operatorname{det}\left(q_{M}\right)\right|_{v}^{2}\right)^{1 / 2} .
\end{aligned}
$$

(We use the inequality $\left\langle x^{a}, x^{a}\right\rangle_{k}=\int_{\mathbb{P}^{N}(\mathbb{C})} h_{\overline{\mathcal{O}(D)}}\left(x^{a}, x^{a}\right) \Omega_{k} \leq 1$ for any $a \in \mathbb{N}_{D}^{N+1}$, which follows from $h \overline{\mathcal{O}(D)}_{k}\left(x^{a}, x^{a}\right) \leq h \overline{\mathcal{O}(D)}_{\infty}\left(x^{a}, x^{a}\right) \leq 1$ on $\mathbb{P}^{N}(\mathbb{C})$ and the facts that $\Omega_{k}>0$ on $\mathbb{P}^{N}(\mathbb{C})$ and $\int_{\mathbb{P}^{N}(\mathbb{C})} \Omega_{k}=1$.)

Then

$$
\left|q_{1} \wedge \cdots \wedge q_{m}\right|_{v} \leq\left\|q_{1} \wedge \cdots \wedge q_{m}\right\|_{k, v}^{\vee}, \quad \forall k \in \mathbb{N} .
$$

By Propositions 2.2 and 2.3, we get

$$
\mathcal{H}_{\text {norm }}(X ; D) \leq \mathcal{H}_{\text {arith }}(X ; D, k), \quad \forall k \in \mathbb{N} \text {. }
$$

By (8), the previous inequality gives

$$
\limsup _{D \rightarrow \infty} \frac{(n+1) !}{D^{n+1}} \mathcal{H}_{\text {norm }}(X ; D) \leq h_{\overline{\mathcal{O}(1)_{k}}}(X), \quad \forall k \in \mathbb{N} .
$$


We know that $\left(h_{k}\right)_{k \in \mathbb{N}}$ converges uniformly to $h_{\infty}$ on $\mathbb{P}^{N}(\mathbb{C})$. Fix $0<\varepsilon<1$. Then there exists a $k_{0} \in \mathbb{N}$ such that, for any $k \geq k_{0}$, we have

$$
(1-\varepsilon)^{2 D} \leq \frac{\left(\max \left(\left|x_{0}\right|_{v}, \ldots,\left|x_{N}\right|_{v}\right)\right)^{2 D}}{\left(\left|x_{0}\right|_{v}^{2 k}+\cdots+\left|x_{N}\right|_{v}^{2 k}\right)^{D / k}} \leq(1+\varepsilon)^{2 D}, \quad \forall x \in \mathbb{P}^{N}(\mathbb{C}), \forall D \in \mathbb{N} .
$$

Thus, for any $k \geq k_{0}, D \in \mathbb{N}_{\geq 1}$ and $a \in \mathbb{N}_{D}^{N+1}$, we get

$$
\left\langle x^{a}, x^{a}\right\rangle_{k} \geq(1-\varepsilon)^{2 D} \int_{\mathbb{P}^{N}(\mathbb{C})} h_{\infty}^{\otimes D}\left(x^{a}, x^{a}\right) \omega_{k}^{N}
$$

We have

$$
\begin{aligned}
\int_{\mathbb{P}^{N}(\mathbb{C})} h_{\infty}^{\otimes D}\left(x^{a}, x^{a}\right) & \omega_{k}^{N} \\
& =\int_{\mathbb{C}^{N}} \frac{\left|z^{2 a}\right|}{\max \left(1,\left|z_{1}\right|, \ldots,\left|z_{N}\right|\right)^{2 D}} \frac{k^{N} \prod_{i=1}^{N}\left|z_{i}\right|^{2(k-1)} \prod_{i=1}^{N} d z_{i} \wedge d \bar{z}_{i}}{\left(1+\sum_{i=1}^{N}\left|z_{i}^{2 k}\right|\right)^{N+1}} \\
& =2^{N} \int_{\left(\mathbb{R}^{+}\right)^{N}} \frac{k^{N} r^{a+k-1}}{\max \left(1, r_{1}, \ldots, r_{N}\right)^{2 D}} \frac{\prod_{i=1}^{N} d r_{i}}{\left(1+\sum_{i=1}^{N} r_{i}^{k}\right)^{N+1}} \\
& =2^{N} \int_{\left(\mathbb{R}^{+}\right)^{N}} \frac{r^{a / k}}{\max _{i}\left(1, r_{1}, \ldots, r_{N}\right)^{D / k}} \frac{\prod_{i=1}^{N} d r_{i}}{\left(1+\sum_{i=1}^{N} r_{i}^{k}\right)^{N+1}} \\
& =2^{N} \sum_{j=0}^{N} \int_{E_{j}} \frac{r^{a / k}}{\max _{i}\left(1, r_{1}, \ldots, r_{N}\right)^{D / k}} \frac{\prod_{i=1}^{N} d r_{i}}{\left(1+\sum_{i=1}^{N} r_{i}\right)^{N+1}},
\end{aligned}
$$

where $E_{j}:=\left\{x \in\left(\mathbb{R}^{+}\right)^{N}: x_{j} \geq 1, x_{l} \leq x_{j}\right.$ for $\left.l=1, \ldots, N\right\}$ for $j=1, \ldots, N$ and $E:=\left\{x \in\left(\mathbb{R}^{+}\right)^{N}: x_{l} \leq 1\right.$, for $\left.l=1, \ldots, N\right\}$. Using the function

$$
\left(\mathbb{R}^{*+}\right)^{N} \rightarrow\left(\mathbb{R}^{*+}\right)^{N}, \quad x=\left(x_{1}, \ldots, x_{N}\right) \mapsto\left(\frac{x_{1}}{x_{j}}, \ldots, \frac{x_{j-1}}{x_{j}}, \frac{1}{x_{j}}, \ldots, \frac{x_{n}}{x_{j}}\right),
$$

for $j=1, \ldots, N$, we can show that there exists a $b^{(j)}=\left(b_{1}^{(j)}, \ldots, b_{N}^{(j)}\right) \in \mathbb{N}^{N}$ such that

$$
\int_{E_{j}} \frac{r^{a / k}}{\max _{i}\left(1, r_{1}, \ldots, r_{N}\right)^{D / k}} \frac{\prod_{i=1}^{N} d r_{i}}{\left(1+\sum_{i=1}^{N} r_{i}\right)^{N+1}}=\int_{E} r^{b^{(j)} / k} \frac{\prod_{i=1}^{N} d r_{i}}{\left(1+\sum_{i=1}^{N} r_{i}\right)^{N+1}} .
$$

We set $b^{(0)}:=a$. Then

$$
\int_{\mathbb{P}^{N}(\mathbb{C})} h_{\infty}^{D}\left(x^{a}, x^{a}\right) \omega_{k}^{N}=2^{N} \sum_{j=0}^{N} \int_{E} r^{b^{(j)} / k} \frac{\prod_{i=1}^{N} d r_{i}}{\left(1+\sum_{i=1}^{N} r_{i}\right)^{N+1}} .
$$


Let $0<\delta<1$, and set $E_{\delta}:=\left\{x \in E: x_{l} \geq \delta\right.$ for $\left.l=1, \ldots, N\right\}$. From (13) and (15), we obtain $\left\langle x^{a}, x^{a}\right\rangle_{k} \geq(1-\varepsilon)^{2 D} 2^{N} \sum_{j=0}^{N} \int_{E_{\delta}} r^{b^{(j)} / k} \frac{\prod_{i=1}^{N} d r_{i}}{\left(1+\sum_{i=1}^{N} r_{i}\right)^{N+1}} \geq(1-\varepsilon)^{2 D} 2^{N}(N+1) \delta^{D / k} \mu_{\delta}$, where $\mu_{\delta}:=\int_{E_{\delta}} \prod_{i=1}^{N} d r_{i} /\left(1+\sum_{i=1}^{N} r_{i}\right)^{N+1}$.

Thus,

$$
\left\langle x^{a}, x^{a}\right\rangle_{k}^{-1} \leq(1-\varepsilon)^{-2 D} \delta^{-D / k} \mu_{\delta}^{-1}, \quad \forall k \geq k_{0}, \forall D \in \mathbb{N}_{\geq 1}, \forall a \in \mathbb{N}_{D}^{N+1} .
$$

Then, for any $k \geq k_{0}$ and $D \geq D_{1}$,

$$
\begin{aligned}
& \left\|q_{1} \wedge \cdots \wedge q_{m}\right\|_{k, v}^{\vee} \leq\left(\sum_{M ; \operatorname{Card}(M)=m}\left(\prod_{b \in M}\langle b, b\rangle_{v, k}^{-1}\right)\right)^{1 / 2}\left|q_{1} \wedge \cdots \wedge q_{m}\right|_{v} \\
& \leq \operatorname{Card}\left\{M \subset \mathbb{N}_{D}^{N+1}: \operatorname{Card}(M)=m\right\}^{1 / 2} \\
& \times(1-\varepsilon)^{-m D} \delta^{-m D / k} \mu_{\delta}^{-m}\left|q_{1} \wedge \cdots \wedge q_{m}\right|_{v} \\
& \leq \operatorname{Card}\left(\mathbb{N}_{D}^{N+1}\right)(1-\varepsilon)^{-m D} \delta^{-m D / k} \mu_{\delta}^{-m}\left|q_{1} \wedge \cdots \wedge q_{m}\right|_{v} \\
& =\left(\begin{array}{c}
N+D \\
N
\end{array}\right)^{1 / 2}(1-\varepsilon)^{-m D} \delta^{-m D / k} \mu_{\delta}^{-m}\left|q_{1} \wedge \cdots \wedge q_{m}\right|_{v} \text {, }
\end{aligned}
$$

where the second line follows by (16).

Therefore,

$\mathcal{H}_{\text {arith }}(X ; D, k)$

$$
\begin{aligned}
\leq \mathcal{H}_{\text {norm }}(X ; D) & +\frac{1}{2} \log \left(\begin{array}{c}
N+D \\
N
\end{array}\right)-D \mathcal{H}_{\text {geom }}(X ; D) \log (1-\varepsilon) \\
& -\frac{D \mathcal{H}_{\text {geom }}(X ; D)}{k} \log \delta-\mathcal{H}_{\text {geom }}(X ; D) \log \mu_{\delta} .
\end{aligned}
$$

By (8), we obtain that

$$
h_{\overline{\mathcal{O}(1)_{k}}}(X) \leq \liminf _{D \rightarrow \infty} \frac{(n+1) !}{D^{n+1}} \mathcal{H}_{\mathrm{norm}}(X ; D)+O(\varepsilon)+\frac{\log \delta}{k} O(1), \quad \forall k \geq k_{0} .
$$

Gathering (12) and (19), we conclude that, for any $0<\varepsilon<1$, there exists a $k_{0} \in \mathbb{N}$ such that

$$
\begin{aligned}
\underset{D \rightarrow \infty}{\limsup } \frac{(n+1) !}{D^{n+1}} \mathcal{H}_{\text {norm }}(X ; D) \leq h_{\overline{\mathcal{O}(1)_{k}}}(X) \\
\quad \leq \liminf _{D \rightarrow \infty} \frac{(n+1) !}{D^{n+1}} \mathcal{H}_{\text {norm }}(X ; D)+O(\varepsilon)+\frac{\log \delta}{k} O(1), \quad \forall k \geq k_{0} .
\end{aligned}
$$


Since $\lim _{k \rightarrow \infty} h_{\overline{\mathcal{O}(1)}_{k}}(X)=h_{\overline{\mathcal{O}(1)} \infty}(X)$ (see for instance [Zhang 1995]) and since $h_{\overline{\mathcal{O}(1)}}(X)=\hat{h}(X)$ (see [Philippon and Sombra 2008, p. 342]), we get

$$
\liminf _{D \rightarrow \infty} \frac{(n+1) !}{D^{n+1}} \mathcal{H}_{\text {norm }}(X ; D)=\limsup _{D \rightarrow \infty} \frac{(n+1) !}{D^{n+1}} \mathcal{H}_{\text {norm }}(X ; D)=\hat{h}(X) .
$$

Thus, we have proved Theorem 1.1.

\section{Acknowledgements}

I am very grateful to Martín Sombra for his helpful conversations and encouragement during the preparation of this paper. I would like to thank Vincent Maillot for his useful discussions.

\section{References}

[Abbes and Bouche 1995] A. Abbes and T. Bouche, "Théorème de Hilbert-Samuel 'arithmétique", Ann. Inst. Fourier (Grenoble) 45:2 (1995), 375-401. MR 96e:14024 Zbl 0818.14011

[Amoroso and David 2003] F. Amoroso and S. David, "Minoration de la hauteur normalisée dans un tore”, J. Inst. Math. Jussieu 2:3 (2003), 335-381. MR 2004m:11101 Zbl 1041.11048

[David and Philippon 1999] S. David and P. Philippon, "Minorations des hauteurs normalisées des sous-variétés des tores", Ann. Scuola Norm. Sup. Pisa Cl. Sci. (4) 28:3 (1999), 489-543. MR 2001a:11109 Zbl 1002.11055

[Gillet and Soulé 1992] H. Gillet and C. Soulé, “An arithmetic Riemann-Roch theorem”, Invent. Math. 110:3 (1992), 473-543. MR 94f:14019 Zbl 0777.14008

[Philippon and Sombra 2008] P. Philippon and M. Sombra, "Hauteur normalisée des variétés toriques projectives”, J. Inst. Math. Jussieu 7:2 (2008), 327-373. MR 2010a:11125 Zbl 1147.11033

[Randriambololona 2001] H. Randriambololona, Hauteurs pour les sous-schémas et exemples d'utilisation de méthodes arakeloviennes en théorie de l'approximation diophantienne, $\mathrm{Ph} . \mathrm{D}$. thesis, Université Paris-Sud, 2001, available at http://www.infres.enst.fr/ randriam/maths/these.pdf.

[Randriambololona 2006] H. Randriambololona, "Métriques de sous-quotient et théorème de HilbertSamuel arithmétique pour les faisceaux cohérents", J. Reine Angew. Math. 590 (2006), 67-88. MR 2007d:14053 Zbl 1097.14020

[Zhang 1992] S. Zhang, "Positive line bundles on arithmetic surfaces", Ann. of Math. (2) 136:3 (1992), 569-587. MR 93j:14024 Zbl 0788.14017

[Zhang 1995] S. Zhang, "Small points and adelic metrics", J. Algebraic Geom. 4:2 (1995), 281-300. MR 96e:14025 Zbl 0861.14019

Communicated by Joseph Silverman

Received 2014-11-19 Revised 2015-09-10 Accepted 2015-10-15

hajli@math.sinica.edu.tw Institute of Mathematics, Academia Sinica,

$6 F$, Astronomy-Mathematics Building,

No. 1, Sec. 4, Roosevelt Road, Taipei 10617, Taiwan 


\section{Algebra \& Number Theory}

msp.org/ant

\section{EDITORS}

MANAGING EDITOR

Bjorn Poonen

Massachusetts Institute of Technology

Cambridge, USA

\author{
EDITORIAL BOARD CHAIR \\ David Eisenbud \\ University of California \\ Berkeley, USA
}

BOARD OF EDITORS

Georgia Benkart

Dave Benson

Richard E. Borcherds

John H. Coates

J-L. Colliot-Thélène

Brian D. Conrad

Hélène Esnault

Hubert Flenner

Sergey Fomin

Edward Frenkel

Andrew Granville

Joseph Gubeladze

Roger Heath-Brown

Craig Huneke

Kiran S. Kedlaya

János Kollár

Yuri Manin

Philippe Michel
University of Wisconsin, Madison, USA

University of Aberdeen, Scotland

University of California, Berkeley, USA

University of Cambridge, UK

CNRS, Université Paris-Sud, France

Stanford University, USA

Freie Universität Berlin, Germany

Ruhr-Universität, Germany

University of Michigan, USA

University of California, Berkeley, USA

Université de Montréal, Canada

San Francisco State University, USA

Oxford University, UK

University of Virginia, USA

Univ. of California, San Diego, USA

Princeton University, USA

Northwestern University, USA

École Polytechnique Fédérale de Lausanne
Susan Montgomery

Shigefumi Mori

Raman Parimala

Jonathan Pila

Anand Pillay

Victor Reiner

Peter Sarnak

Joseph H. Silverman

Michael Singer

Vasudevan Srinivas

J. Toby Stafford

Ravi Vakil

Michel van den Bergh

Marie-France Vignéras

Kei-Ichi Watanabe

Efim Zelmanov

Shou-Wu Zhang
University of Southern California, USA

RIMS, Kyoto University, Japan

Emory University, USA

University of Oxford, UK

University of Notre Dame, USA

University of Minnesota, USA

Princeton University, USA

Brown University, USA

North Carolina State University, USA

Tata Inst. of Fund. Research, India

University of Michigan, USA

Stanford University, USA

Hasselt University, Belgium

Université Paris VII, France

Nihon University, Japan

University of California, San Diego, USA

Princeton University, USA

\section{PRODUCTION}

production@msp.org

Silvio Levy, Scientific Editor

See inside back cover or msp.org/ant for submission instructions.

The subscription price for 2015 is US $\$ 255 /$ year for the electronic version, and $\$ 440 /$ year ( $+\$ 55$, if shipping outside the US) for print and electronic. Subscriptions, requests for back issues and changes of subscribers address should be sent to MSP.

Algebra \& Number Theory (ISSN 1944-7833 electronic, 1937-0652 printed) at Mathematical Sciences Publishers, 798 Evans Hall \#3840, c/o University of California, Berkeley, CA 94720-3840 is published continuously online. Periodical rate postage paid at Berkeley, CA 94704, and additional mailing offices.

ANT peer review and production are managed by EditFLOW ${ }^{\circledR}$ from MSP.

\section{PUBLISHED BY}

- mathematical sciences publishers

nonprofit scientific publishing

http://msp.org/

(C) 2015 Mathematical Sciences Publishers 


\section{Algebra \& Number Theory}

Volume $9 \quad$ No. $10 \quad 2015$

Equivariant torsion and base change

MICHAEL LIPNOWSKI

Induction parabolique et $(\varphi, \Gamma)$-modules

CHRISTOPHE BREUIL

On the normalized arithmetic Hilbert function

MOUNIR HAJLI

SUNE PRECHT REEH

XUANCHENG SHAO

Bounds for Serre's open image theorem for elliptic curves over number fields

DAVIDE LOMBARDO

On 0-cycles with modulus

AMALENDU KRISHNA 